\title{
ARTICLE Eukaryotic elongation factor-2 kinase regulates the cross-talk between autophagy and pyroptosis in doxorubicin-treated human melanoma cells in vitro
}

\author{
Pian Yu ${ }^{1}$, Hai-yan Wang ${ }^{1}$, Min Tian ${ }^{1}$, Ao-xue $\mathrm{Li}^{1}$, Xi-sha Chen ${ }^{1}$, Xin-luan Wang ${ }^{2}$, Yi Zhang ${ }^{3}$ and Yan Cheng ${ }^{1}$
}

\begin{abstract}
Eukaryotic elongation factor-2 kinase (eEF-2K), a negative regulator of protein synthesis, has been shown to play an important role in modulating autophagy and apoptosis in tumor cells under various stresses. In this study, we investigated the regulatory role of eEF-2K in pyroptosis (a new form of programmed necrosis) in doxorubicin-treated human melanoma cells. We found that doxorubicin $(0.5-5 \mu \mathrm{mol} / \mathrm{L})$ induced pyroptosis in melanoma cell lines SK-MEL-5, SK-MEL-28, and A-375 with high expression of DFNA5, but not in human breast cancer cell line MCF-7 with little expression of DFNA5. On the other hand, doxorubicin treatment activated autophagy in the melanoma cells; inhibition of autophagy by transfecting the cells with siRNA targeting Beclin 1 or by pretreatment with chloroquine $(20 \mu \mathrm{mol} / \mathrm{L})$ significantly augmented pyroptosis, thus sensitizing the melanoma cells to doxorubicin. We further demonstrated that doxorubicin treatment activated eEF-2K in the melanoma cells, and silencing of eEF-2K blunted autophagic responses, but promoted doxorubicin-induced pyroptotic cell death. Taken together, the above results demonstrate that eEF-2K dictates the cross-talk between pyroptosis and autophagy in doxorubicin-treated human melanoma cells; suppression of eEF-2K results in inhibiting autophagy and augmenting pyroptosis, thus modulating the sensitivity of melanoma cells to doxorubicin, suggesting that targeting eEF-2K may reinforce the antitumor efficacy of doxorubicin, offering a new insight into tumor chemotherapy.
\end{abstract}

Keywords: eEF-2K; doxorubicin; DFNA5; pyroptosis; autophagy; chloroquine; human melanoma cells; tumor chemotherapy

Acta Pharmacologica Sinica (2019) 40:1237-1244; https://doi.org/10.1038/s41401-019-0222-z

\section{INTRODUCTION}

Pyroptosis, a new form of programmed necrosis, is characterized by the continuous expansion of cells until the cell membranes break down, resulting in the release of cell contents and the activation of a strong inflammatory response [1-3]. Pyroptosis was initially thought to be caspase-1-dependent monocyte death [1]. Subsequently, it was found that caspase-11/4/5, like caspase-1, could also cleave gasdermin D (GSDMD) to generate an $\mathrm{N}$-terminal domain that induces the formation of pores in the cell membrane. Thus, cellular pyroptosis was redefined as a programmed necrosis mediated by gasdermin $[1,2,4-8]$. In 2017, there was a new breakthrough in pyroptosis, when it was revealed that GSDME (DFNA5), another gasderminfamily protein, can be cleaved by activated caspase- 3 to generate a DFNA5-N fragment that targets the plasma membrane to induce pyroptosis $[9,10]$.

Autophagy, a cellular process mainly responsible for degrading long-lived proteins and subcellular organelles [11, 12], occurs at a basal level in all cells and is activated in response to diverse cellular stresses, such as starvation, hypoxia, irradiation, and growth factor deprivation $[13,14]$. Although activation of autophagy leads to autophagic cell death, this form of programmed cell death has also been reported to support cell survival [15-21]. The pro-survival role of autophagy in cancer cells is often associated with negative regulation of apoptosis $[19,20]$. Recently, some relationships between autophagy and caspase-1-mediated pyroptosis have been reported. For instance, autophagy protects infected macrophages and microglia from pyroptosis [21, 22]. Prostatic epithelial cells with higher levels of PDRX3 could promote the initiation and development of benign prostatic hyperplasia by inhibiting autophagy and activating pyroptosis [23]. However, the association between autophagy and pyroptosis in cancer cells and whether autophagy can affect DFNA5-mediated pyroptosis are unknown.

eEF-2K, also called calmodulin-dependent protein kinase III, is a negative regulator of protein synthesis [24]. eEF-2 is the only known substrate of eEF-2K, which promotes ribosomal translocation from the $A$ to the $P$ site, thus improving the movement of the mRNA along the ribosome during translation in eukaryotic tissues [25]. eEF-2 is inactivated when it is phosphorylated on Thr56 by eEF-2K, thus inhibiting protein synthesis [26]. Many

\footnotetext{
${ }^{1}$ Xiangya School of Pharmaceutical Sciences, Central South University, Changsha 410008, China; ${ }^{2}$ Translational Medicine R\&D Center, Institute of Biomedical and Health

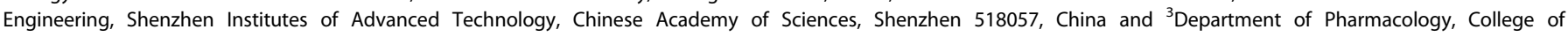
Pharmaceutical Sciences, Soochow University, Suzhou 215000, China

Correspondence: Yi Zhang (zhangyi@suda.edu.cn) or Yan Cheng (chengyan0677@163.com)

These authors contributed equally: Pian Yu, Hai-yan Wang.
}

Received: 2 November 2018 Accepted: 21 February 2019

Published online: 26 March 2019 
studies have observed high eEF-2K expression in various tumor cells [27], and inhibiting the activity of this kinase could reduce the viability of tumor cells [28, 29]. Furthermore, eEF-2Kmediates autophagy in tumor cells in response to various metabolic and therapeutic pressures, such as innutrition [28, 30], growth factor inhibition and chemical irritation [31-33]. This kinase has also been demonstrated to play an important role in modulating autophagy and apoptosis in tumor cells under various stresses; however, the regulatory role of eEF-2K in pyroptosis is unclear.

In the present study, we demonstrated for the first time the relationship between autophagy and DFNA5-mediated pyroptosis, and we found that suppression of eEF-2 kinase enhanced the cytotoxicity of doxorubicin (a broad-spectrum antitumor chemotherapeutic drug) in melanoma cells by facilitating the transition from autophagy to pyroptosis. Therefore, targeting eEF-2K may enhance the antitumor efficacy of doxorubicin, thus offering a new insight into tumor chemotherapy.

\section{MATERIALS AND METHODS}

Cell lines and culture

The SK-MEL-5, SK-MEL-28, and A-375 human melanoma cell lines and the MCF-7 human breast cancer cell line were grown in Dulbecco's modified Eagle's medium (DMEM)/High glucose supplemented with $10 \%$ fetal bovine serum (FBS), which was purchased from Gibco. The cells were maintained at $37^{\circ} \mathrm{C}$ in a humidified atmosphere with $5 \% \mathrm{CO}_{2}$.

Reagents and antibodies

Doxorubicin was purchased from Selleck. Chloroquine (CQ) was purchased from Sigma-Aldrich. Z-VAD-FMK was purchased from Santa Cruz Biotechnology. The rabbit monoclonal antibody recognizing the $\mathrm{N}$-terminal domain of DFNA5 was purchased from Abcam. The rabbit monoclonal antibodies against LC3, Beclin 1, phospho-eEF2 (T56), caspase-3 and cleaved caspase-3 were purchased from Cell Signaling Technologies. Anti- $\beta$-actin was purchased from Proteintech.

shRNA and siRNA Transfection

SK-MEL-5, SK-MEL-28, and A375 cells were seeded in 6-well plates. When the cell density reached $50 \%-60 \%$ confluence, $1 \mathrm{~mL}$ of the lentiviral supernatant containing the lentiviral construct for sh-eEF-2K (which was purchased from Genechem) or the control vector was added to the cells. After 2 days of infection, culture medium containing $0.5 \mu \mathrm{g} / \mathrm{mL}$ puromycin was added to the cells. After 2 additional days, Western blotting was used to validate the eEF-2K-knockdown effect in these cells. The cells were cultured using standard procedures in a humidified incubator at $37^{\circ} \mathrm{C}$ with $5 \% \mathrm{CO}_{2}$ in DMEM media supplemented with $10 \%$ (v/v) FBS (Gibco). The siRNA targeting Beclin 1 was purchased from Ribobio. Non-targeting siRNA was used as a control. Transfection of siRNA was performed according to the manufacturer's protocol. Exponentially growing cells were plated in 6-well tissue culture plates at $1 \times 10^{5}$ cells per well, grown for $24 \mathrm{~h}$, and then transfected with siRNA using Lipofectamine 2000 (Invitrogen) and OPTI-MEM reduced serum medium.

\section{GFP-LC3 punctate assay}

SK-MEL-5, SK-MEL-28, and A-375 cells were transfected with a mixture of GFP-LC3 plasmid and Lipofectamine 2000 (Invitrogen) in no-serum medium for $6 \mathrm{~h}$. On the following day, these cells were treated with a series of doxorubicin concentrations for $24 \mathrm{~h}$. To determine the autophagic response, GFP-LC3 puncta were visualized under an inverted fluorescence microscope.

\section{Western blot analysis}

After treatment, cells were washed with PBS and lysed with RIPA buffer (Beyotime, Haimen, China) supplemented with a protease inhibitor cocktail and a phosphatase inhibitor cocktail (Selleck). The protein concentrations were quantified with a BCA protein assay kit (Beyotime, Haimen, China). The proteins were separated by $12 \%$ SDS-PAGE and then transferred to a PVDF membrane. The PVDF membrane was incubated with primary antibody in 5\% skim milk with Tween 20 for $1 \mathrm{~h}$ at room temperature. The membranes were then incubated with antirabbit or anti-mouse secondary antibody at room temperature for $1 \mathrm{~h}$. Detection was accomplished via chemiluminescence using an $\mathrm{ECL}$ reagent.

Cellular viability assay

Briefly, cells were seeded at $5 \times 10^{3}$ cells per well in 96 -well plates and treated with a series of doxorubicin concentrations for $24 \mathrm{~h}$. At the end of the treatment, $10 \mu \mathrm{L}$ of CCK8 reagent (Biotool) was added to each well, and the cells were incubated for $1 \mathrm{~h}$. At the end of this incubation, the absorbance at $450 \mathrm{~nm}$ wavelength was measured. The effect of doxorubicin on cell viability was determined via a trypan blue exclusion assay. Cells were stained with $0.4 \%$ trypan blue (Wellbiology), and viable cells were counted using a hemocytometer. All experiments were performed independently three times with triplicate samples in each experiment.

\section{Detection of $\mathrm{LDH}$ release}

The levels of lactate dehydrogenase $(\mathrm{LDH})$ in the culture supernatants were measured using the LDH Cytotoxicity Assay Kit from Beyotime according to the manufacturer's instructions. After treatment with a series of doxorubicin concentrations for $24 \mathrm{~h}$, supernatant was transferred to 96-well plates with $120 \mu \mathrm{L}$ in each well. Next, $60 \mu \mathrm{L}$ of $\mathrm{LDH}$ reagent was added to each well, and the cells were incubated for $0.5 \mathrm{~h}$. At the end of this incubation, the absorbance at $490 \mathrm{~nm}$ wavelength was measured.

\section{Statistical analysis}

The difference between the samples treated with doxorubicin alone and samples treated with doxorubicin in combination with $\mathrm{CQ}$ or silencing of eEF-2K were analyzed by $t$-tests. Differences between groups were considered statistically significant at $P<0.05$.

\section{RESULTS}

Doxorubicin induces DFNA5-mediated pyroptosis in melanoma cells

It was reported that cells with high DFNA5 expression underwent pyroptosis upon treatment with chemotherapeutics such as doxorubicin $[9,10]$. First, we examined the DFNA5 expression levels in SK-MEL-5, SK-MEL-28, A-375 human melanoma cells and MCF-7 human breast cancer cells. We observed that DFNA5 was highly expressed in the melanoma cells but was expressed less in MCF-7 cells (Fig. 1a). Thus, we chose SK-MEL-5, SK-MEL-28 and A-375 cells for the subsequent experiments, and we found that doxorubicin-treated cells exhibited characteristic pyroptosis-associated morphology, including cell membrane ballooning; however, we failed to find any pyroptotic morphology in MCF-7 cells (Fig. 1b). To further confirm the stimulatory effect of doxorubicin on pyroptosis, we examined the expression levels of N-DFNA5 in SK-MEL-5, SK-MEL-28 and A-375 cells. NDFNA5 expression was increased in doxorubicin-treated melanoma cells (Fig. 1c). DFNA5 is specifically cleaved by caspase-3; therefore, we measured the protein levels of caspase- 3 and its activated form, cleaved caspase-3. There was an increase in the cleaved caspase-3 level in doxorubicin-treated melanoma cells 
a

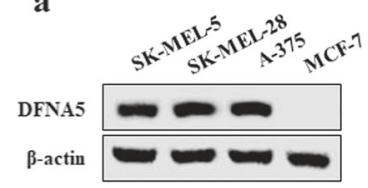

b

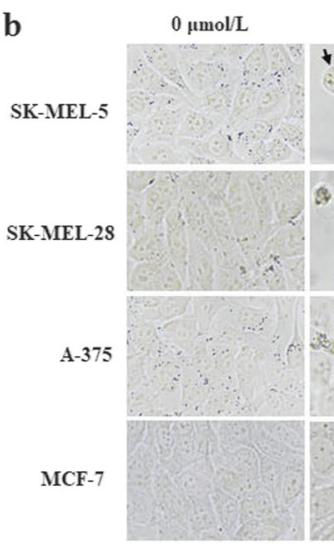

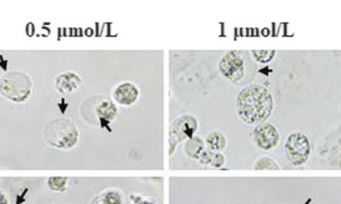

$5 \mu \mathrm{mol} / \mathrm{L}$

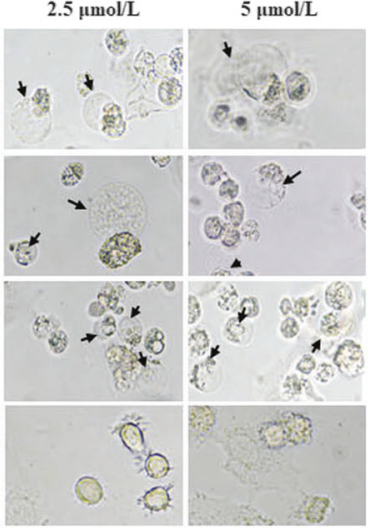

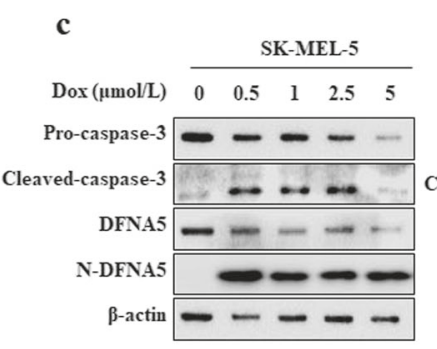

e

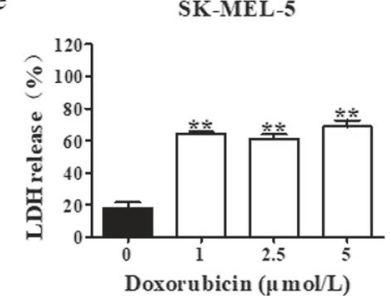

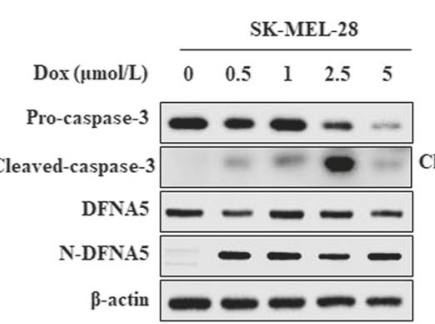

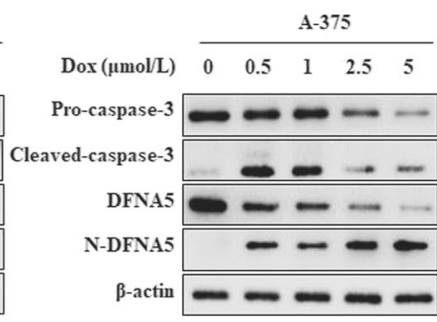

d \begin{tabular}{lllll} 
& \multicolumn{4}{c}{ A-375 } \\
\cline { 2 - 5 } $\operatorname{Dox}(\mu \mathrm{mol} / \mathrm{L})$ & 0 & 1 & 0 & 1 \\
Z-VAD-FMK & - & - & + & +
\end{tabular} Cleaved-caspase- 3

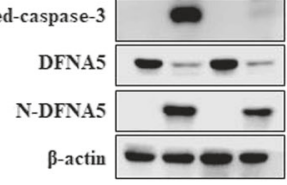

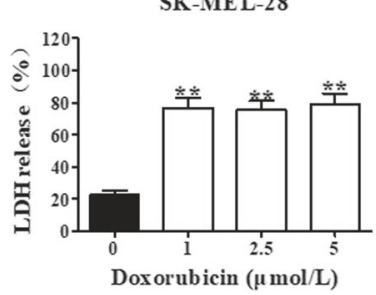

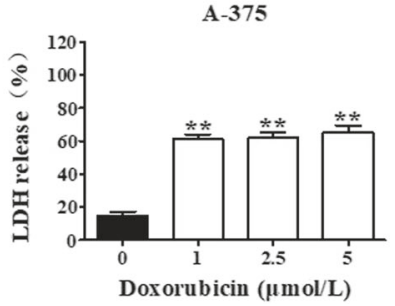

Fig. 1 Doxorubicin induces pyroptosis in melanoma cells. a Differential expression levels in DFNA5 in SK-MEL-5, SK-MEL-28, A-375, and MCF-7 cells. The DFNA5 levels in SK-MEL-5, SK-MEL-28, A-375, and MCF-7 cells were measured by Western blot. $\beta$-actin was used as the loading control. b SK-MEL-5, SK-MEL-28, A-375, or MCF-7 cells were treated with a series of doxorubicin (Dox) concentrations for $24 \mathrm{~h}$. Pyroptotic morphologies were observed at $\times 400$ magnification under an inverted fluorescence microscope. Arrowheads indicate pyroptotic cells. c SKMEL-5, SK-MEL-28, and A-375 cells were treated with a series of doxorubicin (Dox) concentrations for $24 \mathrm{~h}$. The levels of DFNA5, N-DFNA5, caspase-3, and cleaved caspase-3 were measured by Western blot. $\beta$-actin was used as the loading control. d A-375 cells were pretreated with $10 \mu \mathrm{mol} / \mathrm{L}$ Z-VAD-FMK for $1 \mathrm{~h}$, followed by doxorubicin treatment for $24 \mathrm{~h}$. After treatment, the cleaved caspase-3 and N-DFNA5 levels were measured by Western blot. $\beta$-actin was used as the loading control. e At the end of treatment, the LDH levels were measured. Each bar represents the mean \pm SD of triplicate measurements from one of three identical experiments. ${ }^{*} P<0.05$, ${ }^{* *} P<0.01$ vs. the control group

(Fig. 1c). To confirm the relationship between DFNA5 and caspase-3, we treated cells with the Z-VAD-FMK caspase inhibitor and measured the levels of N-DFNA5 and cleaved caspase-3. As shown in Fig. 1d, Z-VAD-FMK attenuated the doxorubicin-dependent increase in the N-DFNA5 level in A-375 cells, indicating that DFNA5 activation was related to caspase-3. Furthermore, we measured the release of $\mathrm{LDH}$, another important indicator of pyroptosis, and we found that the LDH level was increased in doxorubicin-treated cells (Fig. 1e). These results demonstrate that doxorubicin can induce pyroptosis in melanoma cells with high DFNA5 expression, such as SK-MEL-5, SK-MEL-28, and A-375 cells, but cannot induce pyroptosis in cells with low DFNA5 expression, like MCF-7 cells.

Suppression of autophagy enhances pyroptosis in doxorubicintreated melanoma cells

We next examined the induction effect of doxorubicin on autophagy, and we found that the level of LC3 II, an indicator of autophagy, was increased in doxorubicin-treated melanoma cells (Fig. 2a). To further confirm the stimulatory effect of doxorubicin on autophagy, SK-MEL-5, SK-MEL-28, and A-375 cells were transfected with a GFP-LC3 plasmid, followed by doxorubicin treatment. As shown in Fig. $2 b$, there was an increase in the number of GFP-LC3 puncta in the doxorubicin-treated cells. We next investigated the functional relationship between autophagy and pyroptosis in doxorubicin-treated melanoma cells. As shown in Fig. 2c, inhibition of autophagy via transfection with siRNA targeting Beclin1 further increased the N-DFNA5 level compared with doxorubicin alone treatment. To further demonstrate the effect of autophagy on pyroptosis, autophagy was inhibited by using CQ, a chemical inhibitor of autophagy, and we found that combination treatment with CQ further upregulated the levels of N-DFNA5 and cleaved caspase-3 compared with their levels following treatment with doxorubicin alone (Fig. 2d). Furthermore, we found that Z-VAD-FMK could inhibit the increased N-DFNA5 level induced by combination treatment with $C Q$ and doxorubicin (Fig. 2e). The relationship between autophagy and pyroptosis was further assessed by measuring LDH release and trypan blue staining. The percentages of LDH release and trypan blue staining induced by doxorubicin were also markedly increased in cells cotreated with doxorubicin and CQ (Fig. 2f, g). Furthermore, inhibition of autophagy further promoted the doxorubicin- 

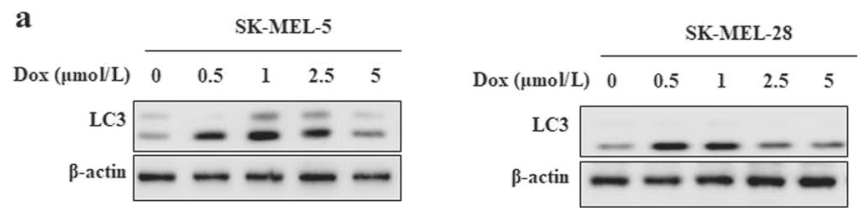

b
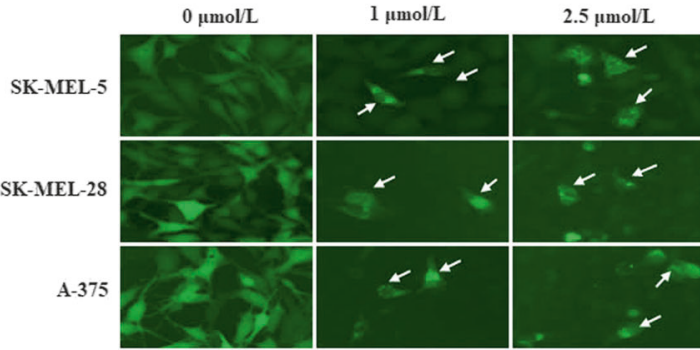

d
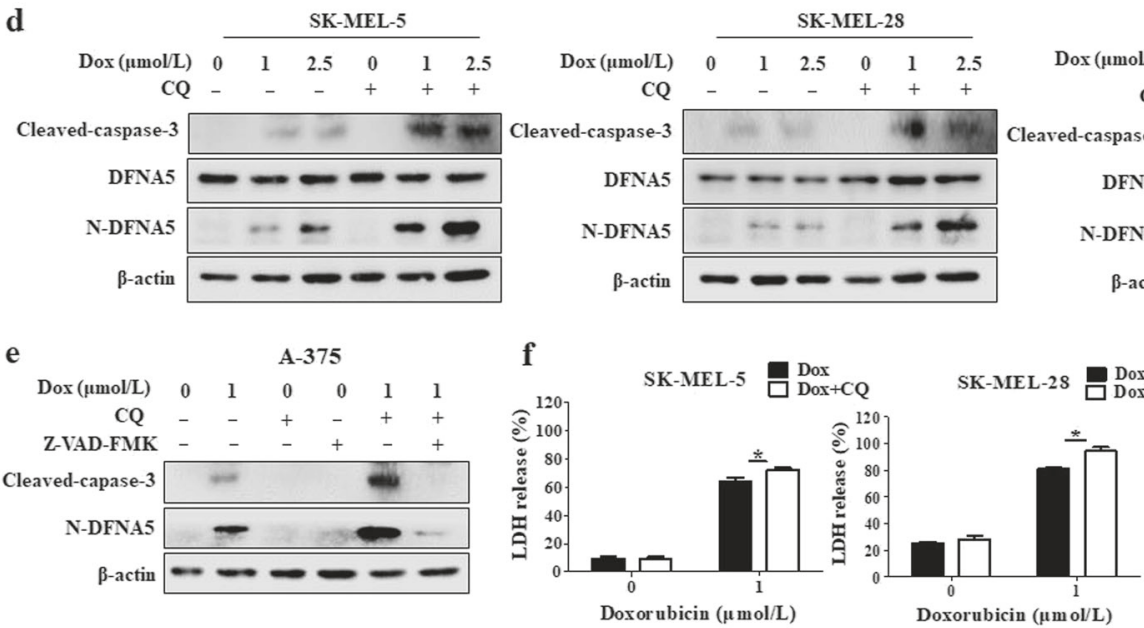

g
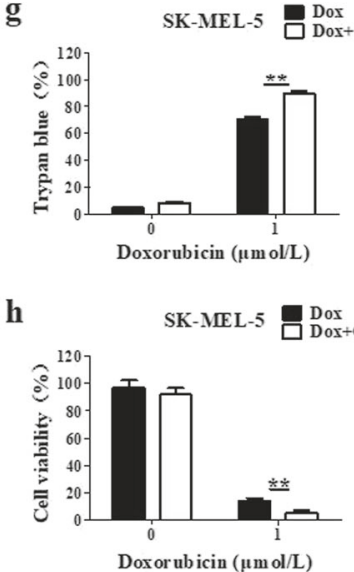
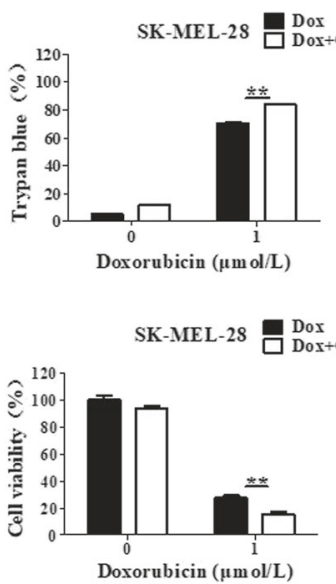

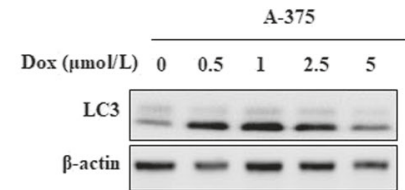

c

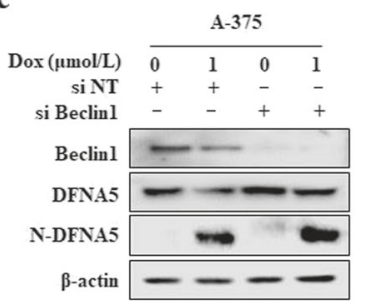

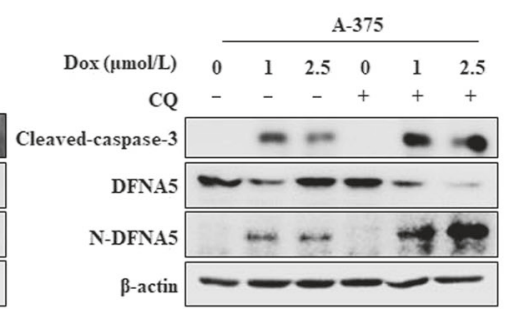


a

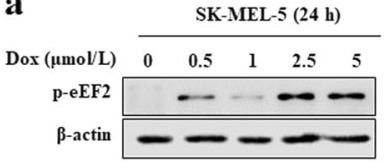

b

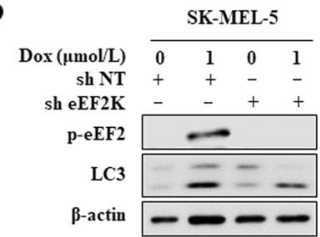

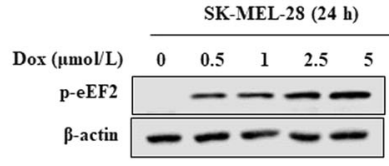
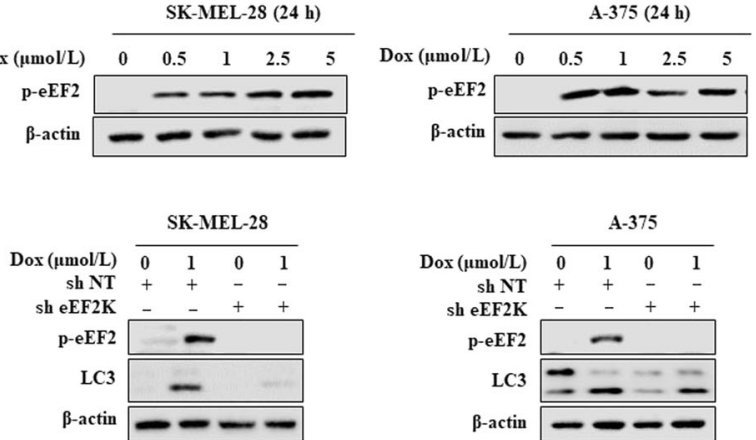

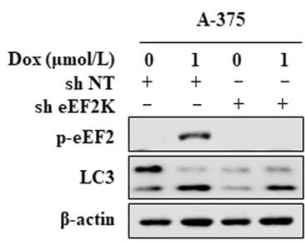

Fig. 3 eEF-2K is involved in doxorubicin-induced activation of autophagy. a SK-MEL-5, SK-MEL-28, or A-375 cells were treated with a series of doxorubicin (Dox) concentrations for $24 \mathrm{~h}$, and the levels phospho-eEF2 (T56) were then measured by Western blot. $\beta$-actin was used as the loading control. b SK-MEL-5, SK-MEL-28, or A-375 cells were treated with $1 \mu \mathrm{mol} / \mathrm{L}$ doxorubicin with or without eEF-2K silencing. The LC3 and phospho-eEF2 (T56) levels were measured by Western blot. $\beta$-actin was used as the loading control

induced reduction in cell viability (Fig. $2 \mathrm{~h}$ ). These results suggest that inhibition of autophagy augmented pyroptosis, thus sensitizing melanoma cells to doxorubicin.

eEF-2K activation is involved in doxorubicin-induced autophagy in melanoma cells

eEF-2K is an important regulator of autophagy under various metabolic and environmental pressures [32, 34, 35]. Therefore, we explored whether this kinase is involved in the activation of autophagy induced by doxorubicin. Doxorubicin-dependent eEF$2 \mathrm{~K}$ activation was indicated by increased eEF-2 phosphorylation (the only known eEF-2K substrate) in SK-MEL-5, SK-MEL-28, and A375 cells (Fig. 3a). To clarify the regulatory effect of eEF-2K in the activation of autophagy by doxorubicin, we silenced eEF-2K and measured autophagic activity. Stable eEF-2K knockdown by lentiviral transduction of short hairpin RNA vectors significantly decreased the LC3-II level in doxorubicin-treated cells (Fig. 3b). These results indicated that the induction of autophagy by doxorubicin is mediated by eEF-2K.

eEF-2K suppression enhances doxorubicin-induced pyroptosis in melanoma cells

We found that eEF-2K is involved in the activation of autophagy and that autophagy prevents pyroptosis in doxorubicin-treated melanoma cells. Thus, we next investigated the role of eEF-2K in the regulation of pyroptosis by comparing the degree of pyroptosis in the doxorubicin-treated melanoma cells with or without eEF-2K silencing. Knockdown of eEF-2K increased pyroptosis in the doxorubicin-treated cells, as indicated by increases in the N-DFNA5 and cleaved caspase-3 levels (Fig. 4a). To further validate the function of eEF- $2 \mathrm{~K}$ in regulating pyroptosis, we measured the LDH release and found that the LDH level was also increased in cells following combined eEF-2K knockdown and doxorubicin treatment (Fig. 4b). The promotive role of eEF-2K knockdown in pyroptosis was further demonstrated by trypan blue staining experiments. Silencing of eEF-2K further increased the percentage of trypan blue-stained cells following doxorubicin treatment (Fig. 4c). We also found that the doxorubicin cytotoxicity was increased when eEF-2K was silenced (Fig. 4d). Taken together, the above results demonstrate that eEF- $2 \mathrm{~K}$ is an intermediary between autophagy and doxorubicin-induced pyroptosis and that eEF-2K inhibition enhanced cellular sensitivity to doxorubicin by promoting pyroptosis.

\section{DISCUSSION}

Autophagy is commonly known to support cell survival; however, the exact roles played by autophagy in response to tumor chemotherapy, what signaling pathways are involved in the regulation of autophagy, and how to expand the application of autophagy are being extensively studied. It has been demonstrated that inhibition of autophagy can enhance the efficacy of antitumor treatments in many different types of cancers by enhancing apoptosis. Furthermore, autophagy has been reported to be linked to caspase-1-mediated pyroptosis. To date, there are no reports on the regulatory relationship between DFNA5mediated pyroptosis and autophagy. Doxorubicin is a broadspectrum antitumor drug that inhibits nucleic acid synthesis to produce a wide range of biochemical effects and strong cytotoxicity. It has been demonstrated that doxorubicin can activate autophagy in cancer cells, including breast cancer cells, hepatocellular carcinoma cells, colon cancer cells, among others [36-38]. Furthermore, it has been previously suggested that the strongly dilated cardiomyopathy induced by doxorubicin is caused by overactivation of autophagy [39-41]. In 2017, it was reported that doxorubicin could induce the DFNA5 cleavage specifically performed by activated caspase-3, ultimately causing pyroptosis. Caspase-3 is a long-recognized hallmark of apoptosis, and it is the DFNA5 expression level that determines the cell death pattern in caspase-3-activated cells. Cells with a high level of DFNA5 undergo pyroptosis upon stimulation by certain agents, including chemotherapeutic drugs; however, cells lacking sufficient DFNA5 undergo apoptosis. Consistent with previous reports, we found that doxorubicin could activate autophagy and pyroptosis in melanoma cells with high DFNA5 expression levels. Importantly, we investigated the relationship between autophagy and pyroptosis, and we found that autophagy antagonized pyroptosis. Our results suggest that inhibition of autophagy not only sensitizes the melanoma cells to doxorubicin treatment by promoting pyroptosis, it may also alleviate the cardiac toxicity induced by this chemotherapeutic drug.

eEF-2K is a unique calcium-dependent enzyme that regulates protein synthesis. Many studies have reported high eEF-2K expression levels in a variety of tumor cell types and that inhibiting eEF-2K activity can reduce the viability of tumor cells. Furthermore, as a central component of the autophagy pathways, eEF-2K is activated and mediates autophagy in tumor cells in response to various metabolic and therapeutic pressures. It has been reported that doxorubicin could induce rapid AMPK activation [42, 43]. Phosphorylation at the ser398 position of eEF-2K by AMPK can control the activity of eEF-2K $[12,44-46]$. These reports suggest that there may be a relationship between eEF-2K activation and doxorubicin and that AMPK is involved in doxorubicin-induced eEF-2K activation. We found that doxorubicin could activate eEF-2K and that knockdown of this kinase inhibited the activity of doxorubicin-induced autophagy. Furthermore, we examined the effects of eEF-2K on pyroptotic cell death and provided new evidence that eEF-2K knockdown enhanced pyroptosis in 
a

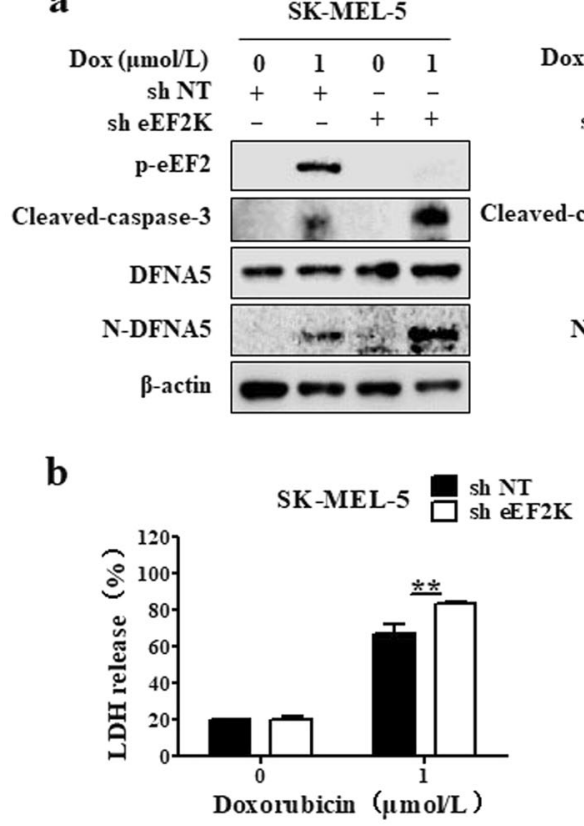

c

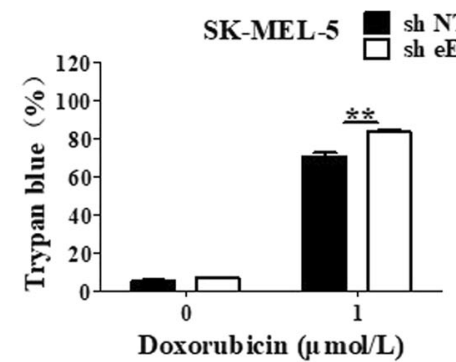

d

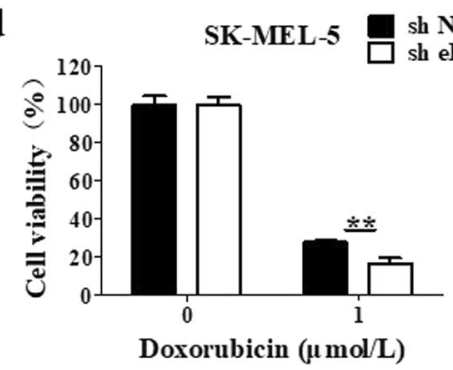

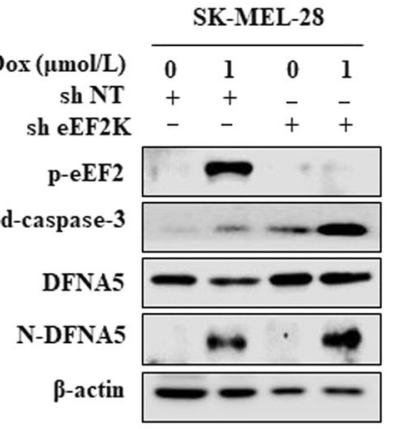
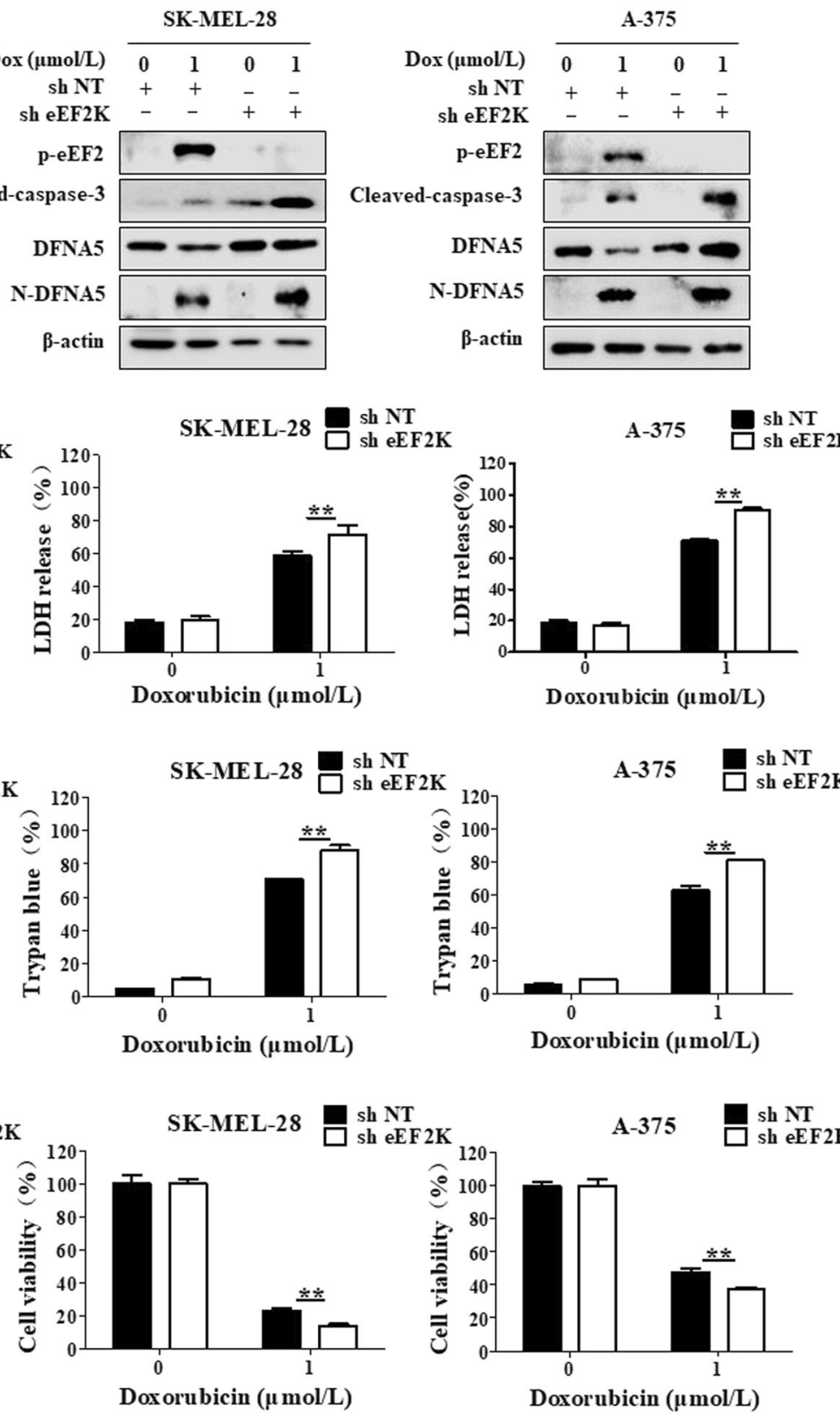

Fig. 4 eEF-2K is a mediator between autophagy and doxorubicin-induced pyroptosis. SK-MEL-5, SK-MEL-28, or A-375 cells were treated with 1 $\mu \mathrm{mol} / \mathrm{L}$ doxorubicin (Dox) for $24 \mathrm{~h}$ with and without eEF-2K silencing. a The phospho-eEF2 (T56), cleaved caspase-3, DFNA5, and N-DFNA5 levels were measured by Western blot. $\beta$-actin was used as the loading control. $\mathbf{b}$ The lactate dehydrogenase (LDH) levels in the culture supernatants were measured using the LDH Cytotoxicity Assay Kit. The results are reported as the mean \pm SD of triplicate measurements; ${ }^{* *} P$ $<0.01, t$-test, doxorubicin vs. doxorubicin + sheEF-2K. c The effects of doxorubicin on cell viability were determined using a trypan blue exclusion assay. The results are reported as the mean \pm SD of triplicate measurements from one of three identical experiments; ${ }^{* *} P<0.01$, doxorubicin vs. doxorubicin + sheEF-2K. d Cell viability was measured with CCK-8 reagent. The results are reported as the mean \pm SD of triplicate measurements; ${ }^{* *} P<0.01, t$-test, doxorubicin vs. doxorubicin + sheEF-2K

doxorubicin-treated melanoma cells. Therefore, inhibiting autophagy and augmenting pyroptosis by inhibiting eEF-2K could enhance the sensitivity of melanoma cells to doxorubicin. On the basis of the results of this study, we believe that eEF-2K could act as an important mediator between autophagy and pyroptosis in cells experiencing chemotherapy, such as doxorubicin, and that it is involved in a previously unidentified and important pathway that regulates the cross-talk between autophagy and pyroptosis to modulate the cellular sensitivity to doxorubicin (Fig. 5). However, the mechanism of how eEF-2K regulates pyroptosis remains unknown. We found the DFNA5 level was increased after eEF-2K knockdown, suggesting that eEF-2K, as a negative regulator of protein synthesis, might affect pyroptosis by inhibiting DFNA5 synthesis. Of course, the precise molecular mechanism by which eEF-2K controls DFNA5 must be investigated.

Based on the results of this study, we confirm that eEF-2K could be important for regulating the switch between autophagy and pyroptosis in doxorubicin-treated melanoma cells. Our findings reveal a previously unidentified and important pathway that regulates the cross-talk between autophagy and DFNA5mediated pyroptosis and modulates cellular sensitivity to doxorubicin. 


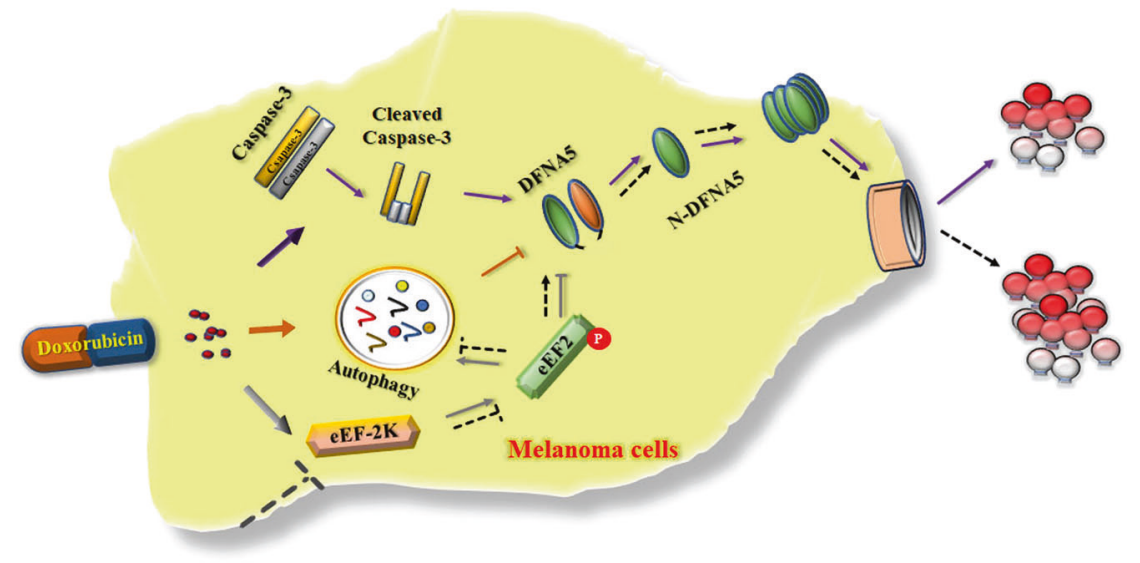

Fig. 5 Proposed role for eEF-2K in the cross-talk between autophagy and DFNA5-mediated pyroptosis in doxorubicin-treated melanoma cells

\section{ACKNOWLEDGEMENTS}

This work was supported by the National Natural Science Foundation of China [grant numbers 81422051, 81472593, 81773749]; Natural Science Foundation of Hunan, China [grant number 2016JJ1020].

\section{AUTHOR CONTRIBUTIONS}

YC and PY conceived and designed the study. PY and HYW performed the experiments. PY, HYW, MT, AXL, and XSC conducted the data analysis. PY drafted the manuscript. YC, YZ, and X.-I.W. revised and approved the manuscript.

\section{ADDITIONAL INFORMATION}

Competing interests: The authors declare no competing interests.

\section{REFERENCES}

1. Bergsbaken T, Fink SL, Cookson BT. Pyroptosis: host cell death and inflammation. Nat Rev Microbiol. 2009;7:99-109.

2. Liu X, Zhang Z, Ruan J, Pan Y, Magupalli VG, Wu H, et al. Inflammasome-activated gasdermin $D$ causes pyroptosis by forming membrane pores. Nature. 2016;535:153-8.

3. Broz P. Immunology: caspase target drives pyroptosis. Nature. 2015;526:642-3.

4. Shi J, Zhao $Y$, Wang $K$, Shi $X$, Wang $Y$, Huang $H$, et al. Cleavage of GSDMD by inflammatory caspases determines pyroptotic cell death. Nature. 2015;526:660-5.

5. Ding J, Wang K, Liu W, She Y, Sun Q, Shi J, et al. Pore-forming activity and structural autoinhibition of the gasdermin family. Nature. 2016;535:111-6.

6. Shi J, Gao W, Shao F. Pyroptosis: gasdermin-mediated programmed necrotic cell death. Trends Biochem Sci. 2017:42:245-54.

7. Aglietti RA, Dueber EC. Recent insights into the molecular mechanisms underlying pyroptosis and gasdermin family functions. Trends Immunol. 2017;38:261-71.

8. Yuan J, Najafov A, Py BF. Roles of caspases in necrotic cell death. Cell. 2016;167:1693-704

9. Wang Y, Gao W, Shi X, Ding J, Liu W, He H, et al. Chemotherapy drugs induce pyroptosis through caspase-3 cleavage of a gasdermin. Nature. 2017;547:99-103.

10. Rogers C, Fernandes-Alnemri T, Mayes L, Alnemri D, Cingolani G, Alnemri ES. Cleavage of DFNA5 by caspase-3 during apoptosis mediates progression to secondary necrotic/pyroptotic cell death. Nat Commun. 2017;8:14128.

11. Levy JMM, Towers CG, Thorburn A. Targeting autophagy in cancer. Nat Rev Cancer. 2017;17:528-42.

12. Cheng $Y$, Ren $X$, Hait WN, Yang JM. Therapeutic targeting of autophagy in disease: biology and pharmacology. Pharmacol Rev. 2013;65:1162-97.

13. Towers CG, Thorburn A. Therapeutic targeting of autophagy. EBioMedicine. 2016;14:15-23.

14. Kundu $M$, Thompson $C B$. Macroautophagy versus mitochondrial autophagy: a question of fate? Cell Death Differ. 2005;12:1484-9.

15. Eskelinen EL. Doctor Jekyll and Mister Hyde: autophagy can promote both cell survival and cell death. Cell Death Differ. 2005;12:1468-72.
16. Wu X, Feng X, Zhao X, Ma F, Liu N, Guo H, et al. Role of Beclin-1-mediated autophagy in the survival of pediatric leukemia cells. Cell Physiol Biochem. 2016;39:1827-36.

17. Paillas S, Causse A, Marzi L, de Medina P, Poirot M, Denis V, et al. MAPK14/ p38alpha confers irinotecan resistance to TP53-defective cells by inducing survival autophagy. Autophagy. 2012;8:1098-112.

18. Matsuzawa Y, Oshima S, Takahara M, Maeyashiki C, Nemoto Y, Kobayashi M, et al. TNFAIP3 promotes survival of CD4 T cells by restricting MTOR and promoting autophagy. Autophagy. 2015;11:1052-62.

19. Houel-Renault L, Philippe L, Piquemal M, Ciapa B. Autophagy is used as a survival program in unfertilized sea urchin eggs that are destined to die by apoptosis after inactivation of MAPK1/3 (ERK2/1). Autophagy. 2013;9:1527-39.

20. Cheng $Y$, Ren $X$, Zhang $Y$, Patel $R$, Sharma $A$, Wu $H$, et al. eEF-2 kinase dictates cross-talk between autophagy and apoptosis induced by Akt Inhibition, thereby modulating cytotoxicity of novel Akt inhibitor MK-2206. Cancer Res. 2011;71:2654-63.

21. Byrne BG, Dubuisson JF, Joshi AD, Persson JJ, Swanson MS. Inflammasome components coordinate autophagy and pyroptosis as macrophage responses to infection. MBio. 2013;4:e00620-12.

22. Kim JY, Paton JC, Briles DE, Rhee DK, Pyo S. Streptococcus pneumoniae induces pyroptosis through the regulation of autophagy in murine microglia. Oncotarget. 2015;6:44161-78.

23. Jiang MY, Han ZD, Li W, Yue F, Ye J, Li B, et al. Mitochondrion-associated protein peroxiredoxin 3 promotes benign prostatic hyperplasia through autophagy suppression and pyroptosis activation. Oncotarget. 2017;8:80295-302.

24. Ryazanov AG, Shestakova EA, Natapov PG. Phosphorylation of elongation factor 2 by EF-2 kinase affects rate of translation. Nature. 1988;334:170-3.

25. Russnes HG, Caldas C. eEF2K-a new target in breast cancers with combined inactivation of p53 and PTEN. EMBO Mol Med. 2014;6:1512-4.

26. Ryazanov AG. Ca2+/calmodulin-dependent phosphorylation of elongation factor 2. FEBS Lett. 1987;214:331-4.

27. Chafouleas JG, Pardue RL, Brinkley BR, Dedman JR, Means AR. Regulation of intracellular levels of calmodulin and tubulin in normal and transformed cells. Proc Natl Acad Sci U S A. 1981;78:996-1000.

28. Ashour AA, Abdel-Aziz AA, Mansour AM, Alpay SN, Huo L, Ozpolat B. Targeting elongation factor-2 kinase (eEF-2K) induces apoptosis in human pancreatic cancer cells. Apoptosis. 2014;19:241-58.

29. Kabil N, Bayraktar R, Kahraman N, Mokhlis HA, Calin GA, Lopez-Berestein G, et al. Thymoquinone inhibits cell proliferation, migration, and invasion by regulating the elongation factor 2 kinase (eEF-2K) signaling axis in triple-negative breast cancer. Breast Cancer Res Treat. 2018;171:593-605.

30. Wu H, Yang JM, Jin S, Zhang $\mathrm{H}$, Hait WN. Elongation factor-2 kinase regulates autophagy in human glioblastoma cells. Cancer Res. 2006;66:3015-23.

31. Wu H, Zhu H, Liu DX, Niu TK, Ren X, Patel R, et al. Silencing of elongation factor-2 kinase potentiates the effect of 2-deoxy-D-glucose against human glioma cells through blunting of autophagy. Cancer Res. 2009;69:2453-60.

32. Cheng Y, Li H, Ren X, Niu T, Hait WN, Yang J. Cytoprotective effect of the elongation factor-2 kinase-mediated autophagy in breast cancer cells subjected to growth factor inhibition. PLoS One. 2010;5:e9715.

33. Guan Y, Jiang S, Yu P, Wen M, Zhang Y, Xiao S, et al. Suppression of eEF-2Kmediated autophagy enhances the cytotoxicity of raddeanin $A$ against human breast cancer cells in vitro. Acta Pharmacol Sin. 2018;39:642-8. 
1244

34. Leprivier G, Remke M, Rotblat B, Dubuc A, Mateo AR, Kool M, et al. The eEF2 kinase confers resistance to nutrient deprivation by blocking translation elongation. Cell. 2013;153:1064-79.

35. Arora S, Yang JM, Kinzy TG, Utsumi R, Okamoto T, Kitayama T, et al. Identification and characterization of an inhibitor of eukaryotic elongation factor 2 kinase against human cancer cell lines. Cancer Res. 2003;63:6894-9.

36. Tan $\mathrm{Q}$, Wang H, Hu Y, Hu M, Li X, Aodengqimuge, et al. SrC/STAT3-dependent heme oxygenase-1 induction mediates chemoresistance of breast cancer cells to doxorubicin by promoting autophagy. Cancer Sci. 2015;106:1023-32.

37. Manov I, Pollak Y, Broneshter R, lancu TC. Inhibition of doxorubicin-induced autophagy in hepatocellular carcinoma Hep3B cells by sorafenib-the role of extracellular signal-regulated kinase counteraction. FEBS J. 2011; 278:3494-507.

38. Fang LM, Li B, Guan JJ, Xu HD, Shen GH, Gao QG, et al. Transcription factor EB is involved in autophagy-mediated chemoresistance to doxorubicin in human cancer cells. Acta Pharmacol Sin. 2017;38:1305-16.

39. Lu L, Wu W, Yan J, Li X, Yu H, Yu X. Adriamycin-induced autophagic cardiomyocyte death plays a pathogenic role in a rat model of heart failure. Int $J$ Cardiol. 2009;134:82-90.
40. Zhang Y, Shi J, Li Y, Wei L. Cardiomyocyte death in doxorubicin-induced cardiotoxicity. Arch Immunol Ther Exp. 2009;57:435-45.

41. Dirks-Naylor AJ. The role of autophagy in doxorubicin-induced cardiotoxicity. Life Sci. 2013;93:913-6.

42. Wang X, Wang X, Chen H, Wu D, Chen J, Wang X, et al. Ghrelin inhibits doxorubicin cardiotoxicity by inhibiting excessive autophagy through AMPK and p38MAPK. Biochem Pharmacol. 2014;88:334-50.

43. Lv X, Yu X, Wang Y, Wang F, Li H, Wang Y, et al. Berberine inhibits doxorubicintriggered cardiomyocyte apoptosis via attenuating mitochondrial dysfunction and increasing Bcl-2 expression. PLoS One. 2012;7:e47351.

44. Browne GJ, Finn SG, Proud CG. Stimulation of the AMP-activated protein kinase leads to activation of eukaryotic elongation factor 2 kinase and to its phosphorylation at a Novel Site, Serine 398. J Biol Chem. 2004;279:12220-31.

45. Horman S, Browne GJ, Krause U, Patel JV, Vertommen D, Bertrand L, et al. Activation of AMP-activated protein kinase leads to the phosphorylation of elongation factor 2 and an inhibition of protein synthesis. Curr Biol. 2002;12:1419-23.

46. Horman S, Beauloye C, Vertommen D, Vanoverschelde J, Hue L, Rider MH. Myocardial ischemia and increased heart work modulate the phosphorylation state of eukaryotic elongation factor-2. J Biol Chem. 2003;278:41970-6. 